\title{
Zr-doped $\mathrm{Al}_{2} \mathrm{O}_{3}$ Grain Boundary and Interfacial Microstructure
}

\author{
Zhenyu Liu
}

Thin Film Technology, Kennametal Inc, 1600 Technology Way, Latrobe, PA 15650, USA

Alumina is one of the key abrasive coating materials for metal-cutting manufacture and semiconductor. It is also a very important thermal barrier coating in the engine system. Alumina coating constitutes micron scale polycrystalline grains and the interaction/connection part of the grains form grain boundaries. Grain boundaries in polycrystalline microstructures are often decorated with dopants intentionally added into the coating systems, or impurities that are acquired during processing and service.

To enhance the performance of the conventional alumina coating and meet the needs for high performance and high speed cutting applications, deliberate tuning of the grains, grain boundary and interfacial structure/chemistry are desired. The alumina CVD coatings were doped or alloyed with transitional metal zirconium and with altering introduction levels. In many metallic and ceramic systems interfaces with various types of segregation of dopant atoms, from sub-monolayer to thick microscopic films, have been observed. The grain boundary/interfaces in the formed and/or doped alumina grains will be modified. It is known that interfaces and the mobility of dopant atoms within an interface play pivotal role in determining the processing and properties of virtually all materials. However, the highly complex nature of the interfaces in solids has been an on-going challenge to link material performance within the internal/intrinsic interface structure and correlated atomic transport mechanism. The nature of the foreign substances profoundly influences materials properties including diffusional transport, interfacial fracture, oxidation, corrosion and creep resistance.

With altering the doping levels by manipulating the dopant introduction, three levels of doping can be predictively achieved (Figure 1): (I) substitution, where dopant atoms substitute of aluminum atoms; it is possible the dopant atoms segregate to grain boundary to induce grain boundary enrichment. (II) substitution plus fine particles, where the fine particle can be in the range of nanoscale as nanodomain in the either grain interior and/or grain boundary (a few $\mathrm{nm}$ and may not be detectable by XRD) [1]; and the last case (III) including substitution plus fine particles and detected another phase by XRD. The transition state of nanoscale particle case, the formed second phase is in very low composition and cannot be detected by XRD. The strengthen mechanism for the first case can be grain boundary enhancement; the second case will combine grain boundary strengthening and fine particles dispersion strengthening mechanism; the strengthening mechanism for the third case will combining with doping, fine particles and the second phase such toughness improvement by embedding dopant phases or composite/nanocomposite, such as $\mathrm{ZrO}_{2}$ in the coating systems [2].

According to the Goldschmidt's Rules on the ionic substitution, the ions of one element can extensively replace those of another in ionic crystals if their radii differ by less than approximately 15\%. (Free substitution can occur if ionic size difference less than 15\%; Limited substitution can occur if ionic size difference is 15 30\%; little or non-substitution can occur if ionic size difference is greater than $30 \%$.) In the doped-alumina coatings, the ionic radius of $\mathrm{Al}^{3+}$ is in the range of $0.53 \sim 0.675 \AA \mathrm{Ti}^{4+}$ is $\sim 0.745 \sim 1.00 \AA ; \mathrm{Zr}^{4+}$ is $\sim 0.73 \sim 0.86 \AA$ and $\mathrm{Hf}^{4+}$ is $\sim 0.72 \sim 0.85 \AA$ depending on their coordination number in the crystal structure, respectively. Considering about the larger $\mathrm{Al}^{3+}$ positions, the ion size differences 
from the early group IVB with aluminum ion can be in the range of $\sim 7 \sim 30 \%$. Thus, it is possible that the doping atoms substitute for the $\mathrm{Al}^{3+}$, where doping atom will occupy the aluminum ion position.

Based on the XRD analysis, it is revealed that the doped-alumina will have different texture structure comparing with corundum alumina. We calculated the texture coefficient of doped-alumina in the different crystal orientations, it is revealed that the doped-alumina will possess altering texture structure. HRTEM study suggested the dopant atoms substitution for aluminium atoms in the formed coatings. The doping process of alumina will modify not only the texture but also the atomic structure of formed grains. High resolution scanning transmission electron microscopy analyses evident that the dopant atoms may distribute at single atom level in the oxide metal ion column by substituting for aluminium ion and enriching at the grain boundary [3,4,5]. The advanced electron microscopy characterization strongly supports that dopant atoms would substitute for the aluminium atoms. Simultaneously, the dopant atoms can segregate and enrich at the grain boundary. The grain boundary engineering can provide enhanced grain interaction and dopant atoms can function as the pinning atoms or provide pinning effect for grain boundary, leading to enhancement of the thermomechanical properties. During the CVD process, the dopant atoms can be introduced into the reactor with alumina grain formations. Therefore, the atom rearrangement and mobility should be more preferable due to the vapor phase of reactants and the reduced energy. It is already accepted that the dopants will profoundly strengthen the alpha- $\mathrm{Al}_{2} \mathrm{O}_{3}$ interface against mechanical deformation.

\section{References:}

[1] Z. P. Li, T. Mori, G. J. Auchterlonie, J. Zou, and J. Drennan, "Nanodomain Formation and Distribution in Gd-doped Ceria", Materials Research Bulletin, 2012, 47, 763-767.

[2] M. Nawa, H. Nakanishi, and Y. Suhero, " $\mathrm{ZrO}_{2}-\mathrm{Al}_{2} \mathrm{O}_{3}$ Composite Ceramic Material and Production Method therefor", US7928028 B2, 2011.

[3] Z. Yu, Q. Wu, J. M Rickman, H. M. Chan, and M. P. Harmer, Scripta Materialia, 2013, 68, 703-706.

[4] N. Shibata, S. D. Findlay, S. Azuma, T. Mizoguchi, T. Yamamoto and Y. Ikuhara, Nature Materials, 2009, 8,654-658.

[5] Z. P. Li, T. Mori, G. J. Auchterlonie, J. Zou, and J. Drennan, Materials Research Bulletin, 2012, 47, 763-767

[6] The authors acknowledge Mr. Tom Nuhfer at Department of Materials Science, Carnegie Mellon University for the help on the TEM observation.

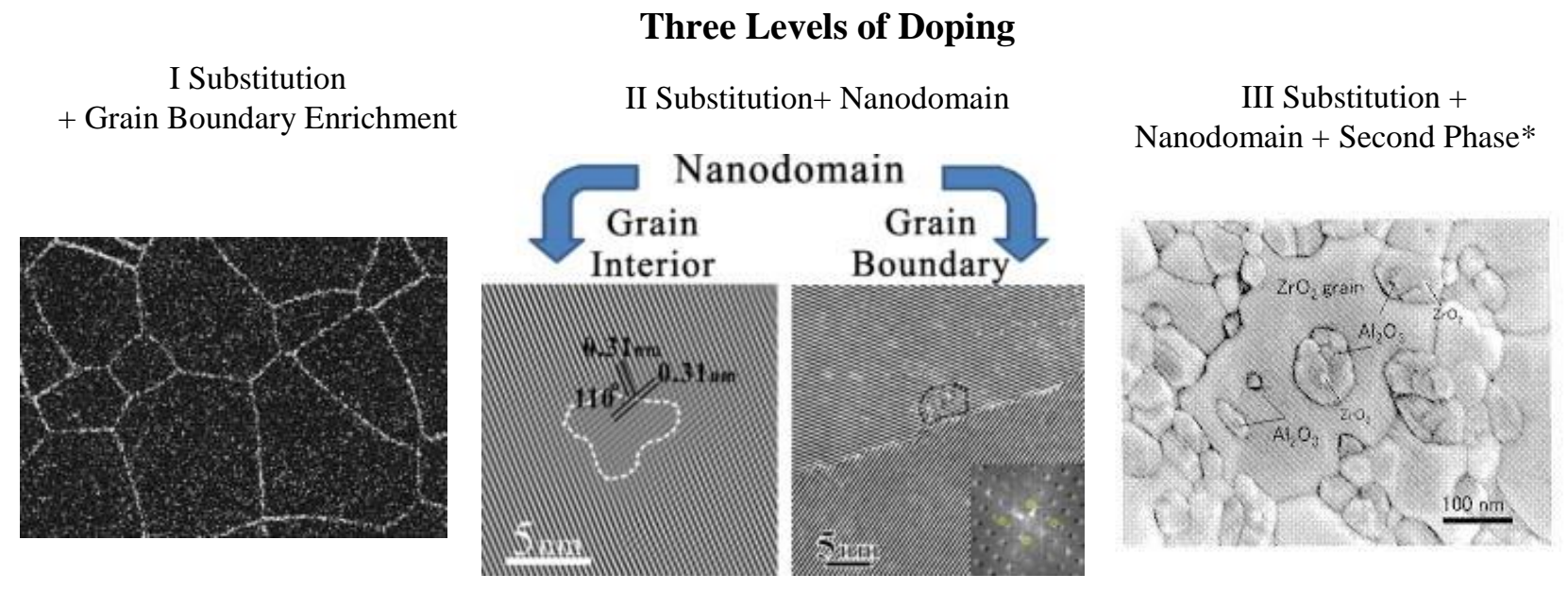

Figure 1. Schematic diagram of three different doping levels: I Substitution plus grain boundary enrichment; II Substitution plus nanodomain; III Substitution, nanodomain and plus second phase*. (* The second phase refers to the detectable phase by XRD). 